\title{
High-Q whispering-gallery mode lasing from nanosphere-patterned GaN nanoring arrays
}

\author{
K. H. Li, Zetao Ma, and H. W. Choi ${ }^{\mathrm{a}}$ \\ Department of Electrical and Electronic Engineering, The University of Hong Kong, Hong Kong
}

(Received 19 October 2010; accepted 26 January 2011; published online 17 February 2011)

\begin{abstract}
A hexagonal-close-packed ordered array of nanorings was fabricated on GaN with a modified nanosphere lithography process. The spheres initially served as etch masks for the formation of closed-packed nanopillars. The spheres were then shrunk and, with a layer of oxide deposited, the roles of the spheres became masks for liftoff. The final etch produced nanorings with wall widths of $140 \mathrm{~nm}$. Photopumped lasing with splitting modes was observed at room temperature, with a low lase threshold of $\sim 10 \mathrm{~mJ} / \mathrm{cm}^{2}$ and high quality factor of $\sim 5000$, via whispering-gallery modes. The resonant frequencies were verified through finite-difference time-domain simulations. (C) 2011 American Institute of Physics. [doi:10.1063/1.3556281]
\end{abstract}

The consumer market is driving increasing demands for blue laser diodes based on the direct wide-band-gap InGaN alloy, an integral optoelectronic component of high-density optical storage systems for entertainment and data storage. Formation of a high-finesse optical cavity is essential for photon confinement in a laser structure. At present, commercially available blue laser diodes are based on the edge-emitting configuration operating in Fabry-Perot mode. Such laser diodes are costly due to the complex and time-consuming fabrication processes. Alternative lasing mechanisms suitable for nitride cavity devices include whispering-gallery mode ${ }^{1,2}$ (WGM) from pivoted GaN-on-Si microdisks $^{3}$ or photoelectrochemically etched undercut microdisk, ${ }^{4,5}$ with reported Q-factors in the range of 1500 4000. Optical confinement via photonic band gap and emission through defect modes in photonic crystal lasers ${ }^{6-8}$ have also been reported, with Q-factors in the range of hundreds.

Traditionally, well-defined cavity structures have relied heavily on electron-beam lithography for precision patterning. Nevertheless, this point-by-point direct-write technique is not suitable for mass production due to high cost and low efficiency. On the other hand, nanosphere lithography ${ }^{9,10}$ (NSL) has been demonstrated as a promising approach toward large area nanopatterning. A monolayer of selfassembled closed-packed nanospheres serves perfectly as etch masks for pattern transfer to the wafer. In our prior works, a wide range of photonic structures and applications have been demonstrated with closed-packed nanopillar arrays formed by NSL, including a photonic crystal LED via the dispersion effect, optically pumped lasing from a nanopillar array at $415.6 \mathrm{~nm}$ via the band-edge effect, ${ }^{11}$ and uv lasing from vertical nanopillar microcavities via the FabryPerot effect. $^{12}$ To widen the flexibility of NSL-enabled structures, a process of producing non-closed-packed nanopillars has also been introduced, ${ }^{13}$ enabling the creation of a photonic band gap. In this work, we take NSL one step further: the generation of closed-packed nanoring arrays using NSL via a dual-step etch process. Optical properties of the nanoring network are fully characterized; the nanorings are demonstrated to function as resonators capable of supporting

\footnotetext{
a) Author to whom correspondence should be addressed. Electronic mail: hwchoi@hku.hk. Tel.: (852) 28592693. FAX: (852) 25598738.
}

whispering-gallery resonant modes. Furthermore, WGM lasing is achieved.

The fabrication of the nanoring arrays evolves from standard processes for forming closed-packed nanosphere monolayers, with additional steps for tweaking the dimensions. Figure 1 illustrates the entire process flow for the fabrication of closed-packed nanoring arrays by NSL. The light-emitting diode (LED) wafer used contains InGaN/GaN multiquantum-wells (MQWs) grown by metal-organic chemical vapor deposition on c-plane sapphire substrate, with an emission center wavelength of $\sim 465 \mathrm{~nm}$, as shown in Fig. 1(a). Silica $\left(\mathrm{SiO}_{2}\right)$ spheres with mean diameters of $1000 \mathrm{~nm}$, dispersed in a combination of de-ionized water and sodium dodecyl sulfate at a volume ratio of 10:1, were dispensed onto the wafer surface using a mechanical micropipette with a volume of $2 \mu \mathrm{l}$. Introduction of a surfactant lowers the surface tension of the colloidal suspension and thus facilitated the spreading of nanosphere to prevent particle agglomeration or aggregation. The excess suspension was then gradually flung off by spin-coating at a constant rotation speed of $700 \mathrm{rpm}$, and a monolayer of spheres was selfassembled on the wafer, as illustrated in Fig. 1(b). The coated monolayer of spheres, which acted as an etch mask, was subsequently transferred to the wafer forming nanopillars as depicted in Fig. 1(c) by inductively coupled plasma
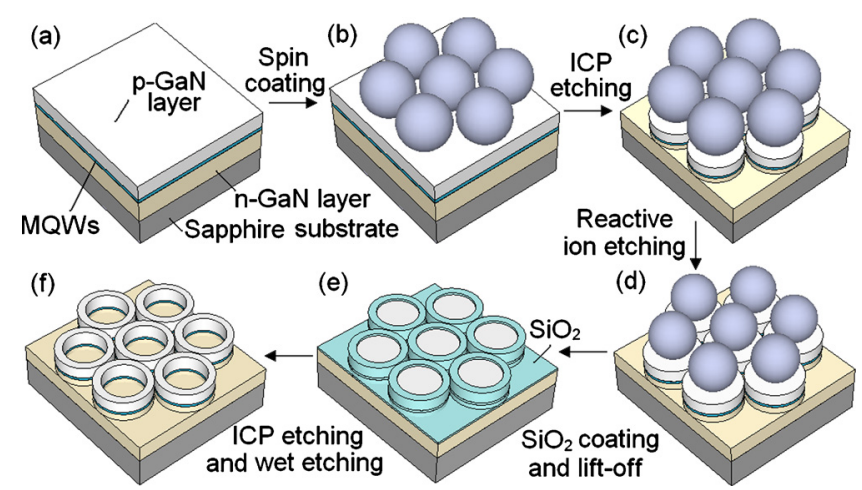

FIG. 1. (Color online) Schematic diagram illustrating the process flow: (a) the starting LED wafer; (b) silica spheres coated on p-GaN layer; (c) pattern transfer to GaN by ICP etching; (d) shrinkage of spheres using RIE etching; (e) $\mathrm{SiO}_{2}$ coating followed by liftoff; (f) removal of $\mathrm{SiO}_{2}$, giving final structure. 

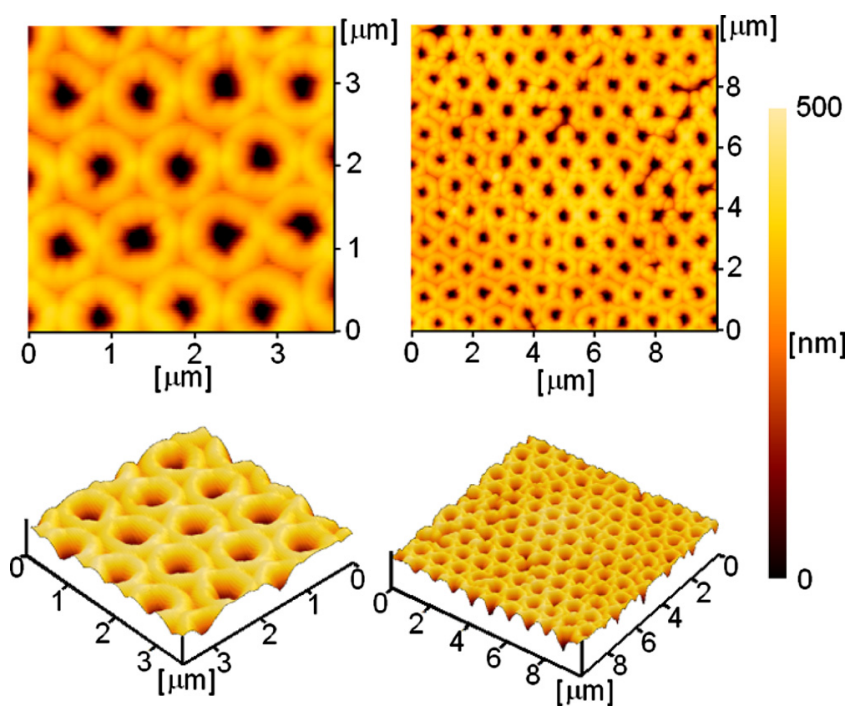

FIG. 2. (Color online) AFM images of the hexagonal-close-packed nanoring arrays.

(ICP) etching using $\mathrm{Cl}_{2}$ as etchant gas at flow rate of 30 sccm. The ICP and platen powers were maintained at 500 and $100 \mathrm{~W}$, while the chamber pressure was fixed at 5 mTorr. The spheres were subsequently shrunk in diameters, as illustrated in Fig. 1(d), although reactive ion etching (RIE) process using $\mathrm{CHF}_{3}$-based plasmas under low rf power conditions to minimize lateral motion; this non-closed-packed sphere array now serves as a liftoff mask. By electron-beam evaporation, a $\mathrm{SiO}_{2}$ layer $(150 \mathrm{~nm})$ was deposited over the entire surface. Following liftoff by sonication in de-ionized water, a ring of $\mathrm{SiO}_{2}$ was left behind at the periphery of the nanopillars as shown in Fig. 1(e); the width of which depends on the extent of the shrinking in the previous step. These oxide rings acted as a mask during the second ICP etch, resulting in the inner-hole region being etched, and the formation of nanorings as illustrated in Fig. 1(f). The residue was removed by immersing the wafer into $10 \%$ hydrofluoric (HF) acid.

Surface morphologies of the nanoring arrays were imaged using a Seiko Nanopics atomic force microscope (AFM), while the optical characteristics were evaluated by time-integrated photoluminescence (PL) at room temperature. A Spectra-Physics diode-pumped solid-state uv laser at $349 \mathrm{~nm}$ emission and pulse duration of $4 \mathrm{~ns}$ at $1 \mathrm{kHz}$ repetition rate was used as an excitation source. The focused beam was incident nearly perpendicular to the sample surface with a spot diameter of around $150 \mu \mathrm{m}$. The PL signal was collected at $30^{\circ}$ to the normal with an optical fiber bundle and the signal coupled to a spectrometer comprising an Acton SP2500A $500 \mathrm{~mm}$ spectrograph and a Princeton Instrument PIXIS open-electrode charge-coupled device, which offered optical resolutions better than $0.04 \mathrm{~nm}$.

The assembled nanoring array is shown in the AFM images in Fig. 2, revealing rings with internal diameter of $720 \mathrm{~nm}$, wall width of $140 \mathrm{~nm}$, and height of $500 \mathrm{~nm}$. The arrays are regularly closed-packed in a hexagonal arrangement. PL spectra of the nanorings at increasing excitation densities are plotted in Fig. 3(a). Under low excitation, the spectrum exhibits weak spontaneous emission from MQWs at $\lambda=460 \mathrm{~nm}$ with full width at half-maximum (FWHM) of $34 \mathrm{~nm}$. As the excitation energy density exceeds the
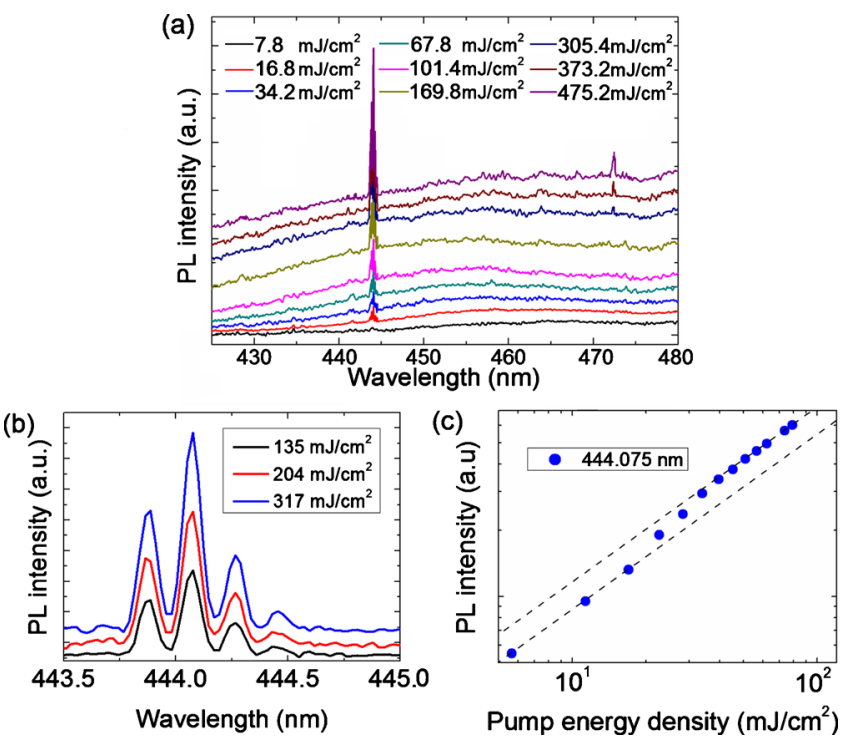

FIG. 3. (Color online) (a) Room temperature PL spectra of nanoring array under varying excitation energy density. (b) An enlarged view of the PL spectrum near the lasing frequency. (c) PL intensity of the lasing peak at $444.075 \mathrm{~nm}$ as a function of pump energy density in logarithmic scale.

threshold of $16.8 \mathrm{~mJ} / \mathrm{cm}^{2}$, a sharp spectral peak emerged at $444 \mathrm{~nm}$; beyond $305.4 \mathrm{~mJ} / \mathrm{cm}^{2}$, a second spectral peak at $472 \mathrm{~nm}$ was observed. Zooming in on the spectrum, three satellite spectral peaks at 443.88, 444.08, and $444.26 \mathrm{~nm}$ with FWHM as low as $0.09 \mathrm{~nm}$ could be resolved as evident in Fig. 3(b); their quality factors, defined by $Q=f / \Delta f$, are evaluated as 4455, 5025, and 4660, respectively, where $f$ is the resonant frequency and $\Delta f$ is the FWHM.

Figure 3(c) plots intensities of the $444.075 \mathrm{~nm}$ lasing peak as a function of excitation energy density in a logarithmic scale. Nonlinear increases in PL intensities were observed beyond the extracted threshold value of $\sim 10 \mathrm{~mJ} / \mathrm{cm}^{2}$. To characterize the optical cavity, the spontaneous emission coupling factor $\beta$, which represents a figure of merit for the cavity, is evaluated. $\beta$, defined as the fraction of total spontaneous emission from the source that is coupled into a cavity mode, can be estimated from the ratio of output intensities below and above the lasing threshold. ${ }^{14}$ In practice, it is always less than unity due to the concurrent emission of nonresonant modes. $\beta$ of spectral peak at $444.075 \mathrm{~nm}$ was estimated to be 0.75 . The Purcell factor, which quantifies the ratio of spontaneous emission rate in a resonant cavity mode to the spontaneous emission rate in the absence of cavity, can be calculated by the following equation ${ }^{15}$ :

$$
F_{p}=\beta /(1-\beta),
$$

from which $F_{p}$ was estimated to be 3 . Being greater than unity, it is implied that the spontaneous emission rate has indeed been enhanced by the cavity and that excitons can radiate much faster in the cavity than in free space. To identify the position of WGM lasing frequency, the resonant frequency and mode spacing for a ring cavity were calculated from the equations $2 \pi R n=m \lambda$ and $\Delta \lambda_{\mathrm{WG}}=\lambda^{2} / 2 \pi R n$, where $n$ is the refractive index of $\mathrm{GaN}, R$ is the ring radius, and $m$ is the mode number. The calculated mode at $m=17$ corresponds to the observed lasing peak at $444 \mathrm{~nm}$, while the mode spacing is evaluated to be in the range of $26-29 \mathrm{~nm}$, correlating well with the PL spectra. 

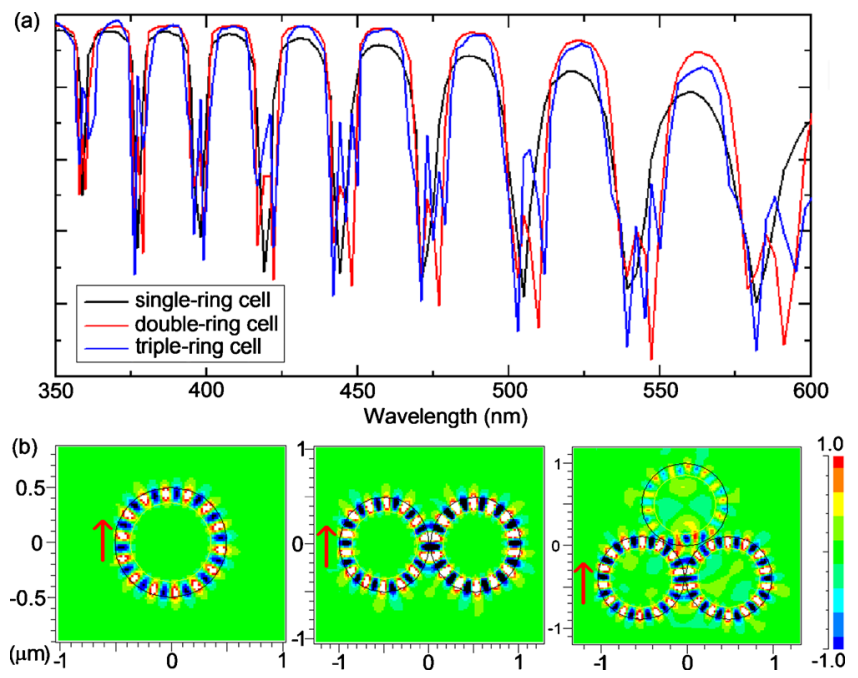

FIG. 4. (Color online) (a) FDTD simulated transmittance plot showing resonant modes for three different cell structures; (b) strong optical coupling occurs between adjacent rings in an array.

The mechanism for stimulated emission from the nanoring array is investigated. Fabry-Perot mode lasing requires the existence of a longitudinal cavity for light confinement. $^{12,17}$ Without mirror coatings such a cavity would not exist and Fabry-Perot type lasing can thus be ruled out. On the other hand the circular geometry of the nanostructures hints at the possibility of WGM resonance in the planar direction. Indeed, nanoring cavities are known to support optical confinement modes which are guided by total internal reflections circling around the peripheries of the rings.

To verify resonant properties of the nanoring cavity, finite-difference time-domain (FDTD) simulations were performed on a ring structure with internal diameter of $720 \mathrm{~nm}$ and wall width of $140 \mathrm{~nm}$. To yield better accuracy, the FDTD mesh size is set to less than $1 / 20$ of wavelength. The computational grid contains $150 \times 150$ nodes over an area of $3 \times 3 \mu \mathrm{m}^{2}$, and the time step is set to $0.045 \mathrm{fs}$, sufficiently small to satisfy the Courant stability condition. The simulated transmittance spectra for three different cell structures are shown in Fig. 4(a), which correlate well with the calculated resonant frequencies and mode spacing, and also the lasing positions from the PL spectra. Compared to a singlering cell, the optical confinement efficiencies of the doublering and triple-ring cells are increased, implying that multicoupled ring arrays have stronger optical confinement effects. Apart from such enhancements, splitting of the resonant modes is also introduced for the multicoupled ring structure. ${ }^{16}$ As individual ring resonators possess the same optical path length, no phase-shift changes exist within a single ring. On the other hand, due to strong optical coupling between rings, there exists round-trip phase shifts when light passes through the couplers, thus resulting in the optical splitting effect. ${ }^{18}$ It explains why multiple peaks appear in the vicinity of $444 \mathrm{~nm}$. Additionally, sidewall-roughness induced backreflection into the counterpropagating modes may yet be another mode-splitting factor. ${ }^{19}$ The performance of traveling-wave resonators is affected when the forward- and backward-propagation modes are phase-matched. Such contradirectional coupling leads to annihilation of directionality of a traveling-wave resonator and causes the resonant peak to split into multiple modes.

The simulated results shown in Fig. 4(b) illustrate the formation of standing waves in single-ring structure when a continuous wave at $444 \mathrm{~nm}$ was injected at the leftmost position indicated by the red arrow. At the resonant frequencies, photons are effectively confined within the ring structures establishing a whispering-gallery mode for the single-ring cavity. For the double-ring and triple-ring cells, highintensity standing waves are set up across the multicoupled ring structure, indicating that strong light coupling between adjacent rings occurs, and a resonance mode was established due to constructive interference. The ring can thus serve as a resonator and interact further with adjacent rings. This supports the postulation of WG mode lasing observed from PL spectra.

In summary, we have demonstrated the fabrication of nanoring arrays by a modified NSL process. The nanoring network functioned as resonators which support whisperinggallery modes. Moreover, room temperature lasing has been observed, with lasing spectral peaks at 443.88, 444.075, and $444.26 \mathrm{~nm}$. Their corresponding Q-factors were evaluated to be 4455,5025 , and 4660, respectively. Optical resonance of the nanoring structure was further verified with FDTD simulations, which was found to correlate well with the frequencies of lasing.

This work was supported by a GRF grant of the Research Grant Council of Hong Kong (Project No. HKU 7118/ 09E).

${ }^{1}$ S. L. McCall, A. F. J. Levi, R. E. Slusher, S. J. Pearton, and R. A. Logan, Appl. Phys. Lett. 60, 289 (1992)

${ }^{2}$ K. C. Zeng, L. Dai, J. Y. Lin, and H. X. Jiang, Appl. Phys. Lett. 75, 2563 (1999).

${ }^{3}$ H. W. Choi, K. N. Hui, P. T. Lai, P. Chen, X. H. Zhang, S. Tripathy, J. H. Teng, and S. J. Chua, Appl. Phys. Lett. 89, 211101 (2006).

${ }^{4}$ A. C. Tamboli, E. D. Haberer, R. Sharma, K. H. Lee, S. Nakamura, and E. L. Hu, Nat. Photonics 1, 61 (2007).

${ }^{5}$ A. C. Tamboli, M. C. Schmidt, A. Hirai, S. P. DenBaars, and E. L. Hu, Appl. Phys. Lett. 94, 251116 (2009).

${ }^{6}$ H. Matsubara, S. Yoshimoto, H. Saito, Y. Jianglin, Y. Tanaka, and S. Noda, Science 319, 445 (2008).

${ }^{7}$ L.-M. Chang, C.-H. Hou, Y.-C. Ting, C.-C. Chen, C.-L. Hsu, J.-Y. Chang, C.-C. Lee, G.-T. Chen, and J.-I. Chyi, Appl. Phys. Lett. 89, 071116 (2006)

${ }^{8}$ Y.-S. Choi, K. Hennessy, R. Sharma, E. Haberer, Y. Gao, S. P. DenBaars, S. Nakamura, E. L. Hu, and C. Meier, Appl. Phys. Lett. 87, 243101 (2005).

${ }^{9}$ J. C. Hulteen and R. P. Van Duyne, J. Vac. Sci. Technol. A 13, 1553 (1995).

${ }^{10}$ C. L. Haynes and R. P. Van Duyne, J. Phys. Chem. B 107, 7426 (2003).

${ }^{11}$ W. Y. Fu, K. Y. Y. Wong, and H. W. Choi, J. Appl. Phys. 107, 063104 (2010).

${ }^{12}$ R. Chen, H. D. Sun, T. Wang, K. N. Hui, and H. W. Choi, Appl. Phys. Lett. 96, 241101 (2010).

${ }^{13}$ K. H. Li and H. W. Choi, J. Appl. Phys. 109, 023107 (2011).

${ }^{14}$ H. Takashima, H. Fujiwara, S. Takeuchi, K. Sasaki, and M. Takahashi, Appl. Phys. Lett. 92, 071115 (2008).

${ }^{15}$ T. C. Lu, C. C. Kao, H. C. Kuo, G. S. Huang, and S. C. Wang, Appl. Phys Lett. 92, 141102 (2008).

${ }^{16}$ R. Guider, N. Daldosso, A. Pitanti, E. Jordana, J. M. Fedeli, and L. Pavesi, Opt. Express 17, 20762 (2009).

${ }^{17}$ J. L. Jewell, J. P. Harbison, A. Scherer, Y. H. Lee, and L. T. Florez, IEEE J. Quantum Electron. 27, 1332 (1991).

${ }^{18}$ D. D. Smith, H. Chang, K. A. Fuller, A. T. Rosenberger, and R. W. Boyd, Phys. Rev. A 69, 063804 (2004).

${ }^{19}$ B. E. Little, J. P. Laine, and S. T. Chu, Opt. Lett. 22, 4 (1997) 